\title{
Demandas institucionais e de cuidado no gerenciamento de enfermeiros em um pronto socorro
}

\author{
Institutional and care demands in the management of nurses in an emergency unit
}

Demandas institucionales e de cuidado en la gerencia de enfermeros en una unidad de urgencia

\author{
Juliana Helena Montezelli', Aida Maris Peres', Elizabeth Bernardino' \\ 'Universidade Federal do Paraná. Programa de Pós-graduação em Enfermagem. \\ Grupo de Pesquisas em Políticas, Gestão e Práticas de Saúde. Curitiba, PR
}

Submissão: 21/01/2010

Aprovação: 03/10/2010

\section{RESUMO}

Objetivo: Caracterizar as atividades gerenciais do enfermeiro em um pronto-socorro. Método: Pesquisa Qualitativa realizada de fevereiro a abril de 2009 por entrevista semi-estrututrada com oito enfermeiros de um pronto-socorro em um hospital-escola de Curitiba, PR, Brasil. Os dados foram submetidos à análise de conteúdo. Resultados: Emergiram duas categorias: Gerência voltada para atender às demandas institucionais, Que salienta a ênfase às atividades burocráticas exigidas do enfermeiro por parte do hospital; e Gerência voltada para atender às demandas do cuidado de enfermagem, Que prioriza o cuidado como foco da atividade gerencial. Conclusão: $\mathrm{O}$ estudo atingiu seu objetivo e coadunou os achados da literatura de Que a cisão entre cuidado e gerenciamento não encontra eco na atuação do enfermeiro em um pronto-socorro.

Descritores: Enfermagem; Enfermagem em emergência; Pesquisa em administração de enfermagem; Gerenciamento de prática profissional.

\section{ABSTRACT}

Objective: To characterize the registered nurse's management activities in an emergency department. Method: Qualitative research, implemented from February to April 2009 by a semi-structured interview with eight nurses from an emergency department at a university hospital in Curitiba, PR. Brazil. The data was submitted to content analyses. Results: Two categories emerged: Management focused on meeting the institutional demands that emphasizes the Registered Nurses' burocratic activities reouired by the hospital; and Management focused on meeting the nursing care demands that prioritizes the care as the main management activity. Conclusion: The study reached its objective and joined the literature findings that the division between care and management does not match with the registered nurse's performance at an emergency department.

Key words: Nursing; Emergency nursing; Nursing administration research; Practice management.

\section{RESUMEN}

Objetivo: Caracterizar las actividades gerenciales del enfermero en la sala de emergencia. Método: Pesquisa cualitativa realizada de febrero a abril de 2009 por entrevista semi-estructurada con ocho enfermeros de una sala de emergencia en un hospital-escuela de Curitiba, PR, Brasil. Los datos fueron sometidos a análisis de contenido. Resultados: Emergieron dos categorías: Gerencia dirigida para atender las demandas institucionales eue muestra el énfasis de las actividades burocráticas exigidas del enfermero por parte del hospital; $y$ Gerencia dirigida para atender las demandas del cuidado de enfermería, Que prioriza el cuidado como foco de la actividad gerencial. Conclusión: El estudio cumplió su objetivo e incorporó lo que se encontró de la literatura de que la división entre cuidado y gerencia no encuentra eco en la actuación del enfermero en la sala de emergencia.

Desciptores: Enfermería; Enfermería de urgencia; Investigación en administración de enfermería; Manejo práctico.

\footnotetext{
AUTOR CORRESPONDENTE Juliana Helena Montezelli. Rua Itajubá, 644, apto 302. CEP 81070-190. Portão, PR

E-mail: jhmontezelli@hotmail.com
} 


\section{INTRODUÇÃO}

As atividades de enfermagem Que constroem o seu processo de trabalho estão pautadas em diversos subprocessos interligados. Estes se estruturam com base nas práticas cuidativas e administrativas ou gerenciais e, para execução das suas ações, sabe-se Que desde a sua concepção como profissão, o parcelamento do trabalho entre os diferentes membros da equipe teve na gerência o elo de articulação das atividades e de sua integração ao processo de trabalho em saúde como um todo.

Como característica marcante do processo de trabalho da enfermagem tem-se o fato de Que este deve ser desenvolvido a partir da realização hologramática de diversos subprocessos, descritos por diferentes autores como inerentes ao assistir, administrar/gerenciar, ensinar e pesquisar ${ }^{(1)}$.

Além destes, a participação política também deve ser considerada uma esfera Que compõe o processo de trabalho da profissão. Ela permeia todos os demais subprocessos, possui como objeto a força de trabalho em enfermagem e sua representatividade, com finalidade de conquistar melhores condições para operar os outros processos e seu produto é poder, reconhecimento social e conQuista de condições favoráveis para operar os processos de trabalho ${ }^{(2)}$.

Percebemos, então, Que o gerenciamento é um subprocesso complementar Que constitui o processo de trabalho do enfermeiro. Assim, deve-se reconhecer o cuidado como foco possível e necessário de ser gerenciado dentro das instituições em uma dimensão Que extrapole o tecnicismo e incorpore o conhecimento e atitudes de ordem racional e sensível.

Mesmo diante disso, o exercício da função gerencial pelo enfermeiro em nosso país permanece como uma Questão mesclada de dúvidas, desentendimentos e incompreensões. $\mathrm{O}$ fato de o enfermeiro limitar-se a atividades administrativas em seu trabalho é considerado polêmico na profissão, tornando-se evidente a discrepância entre o Que se espera do enfermeiro na visão teórica de enfermagem e o Que se verifica ser sua atuação cotidiana nas instituições de saúde ${ }^{(3)}$.

A divisão do trabalho na enfermagem, desde a sua profissionalização, traz o enfermeiro como figura responsável pela atuação administrativa nos serviços de saúde. Sua participação na gerência instiga a compreensão do significado desta prática, tendo em vista a evolução da enfermagem como profissão. Porém, o entrelaçamento entre cuidar e gerenciar sempre se mostrou como um desafio, tendo sido mais enfatizado a partir da década de 1990 tanto no aspecto da formação do enfermeiro como na atuação de lideranças de enfermagem ${ }^{(4)}$.

Notamos a partir das considerações exaradas pelos autores aQui mencionados, Que os diferentes subprocessos compositores do processo de trabalho da enfermagem precisam ser articulados na prática profissional, com foco no cuidado. Todavia, há uma divergência entre essa idéia e as demandas das instituições de saúde em Que se inserem os enfermeiros, pois para a instituição, as funções administrativas desempenhadas por esses profissionais devem ser destinadas à produção com vistas a atender aos princípios capitalistas e não necessariamente à assistência de enfermagem.

No entanto a lógica das instituições hospitalares estarem fundamentadas majoritariamente no modelo de racionalização do trabalho tem se mostrado insuficiente nos tempos pós-modernos pelo conhecimento gerado e as análises realizadas neste século(4).

Esta disparidade de compreensão de atividade gerencial do enfermeiro colabora para Que este profissional possua uma tendência ao afastamento do cuidado. Existe a priorização de tarefas, muitas vezes impostas pelas instituições de saúde, em detrimento à assistência de enfermagem, a Qual geralmente é delegada a outras categorias $^{(5)}$

Reportando-nos ao contexto emergencial, por sua vez, assim como em todas as dimensões de atuação da enfermagem, não há espaço para imprecisões no gerenciamento, pois os enfermeiros da unidade de emergência devem aliar controle do tempo à fundamentação teórica, à capacidade de liderança, ao discernimento, à iniciativa, à habilidade de ensino, à maturidade e à estabilidade emocional $^{(6)}$

Ao longo da atuação profissional em um serviço de emergência hospitalar, deparamo-nos com constantes dificuldades em direcionar a atividade gerencial para o cuidado de enfermagem. Cotidianamente é possível verificar a incoerência entre o gerenciamento mobilizado em prol do cuidado com aQuelas ações exigidas pela organização, Que almejam basicamente atos burocráticos.

Essa percepção empírica converge com colocações da literatura correlata, Que salienta Que tais divergências decorrem das necessidades de atendimento à demanda e produtividade das instituições de saúde, Que representam o mercado de trabalho em enfermagem. Esta ênfase na dimensão técnica do 'fazer' contradiz a ação crítico-reflexiva do enfermeiro pretendida pelas várias dimensões do ensino e pelas políticas de saúde ${ }^{(7)}$.

É preciso redimensionar o foco da administração em enfermagem, pois o hospital visto como produto da Revolução Industrial e Científica incorporou a organização racional do trabalho como exclusiva, sem adição da esfera expressiva. O enfermeiro, por conseguinte, muitas vezes também formado nesta mesma lógica, conformou seu modo de gerenciar cotidiano fazendo cumprir normas e rotinas que se cristalizam ao longo do tempo, truncando as muitas possibilidades de inserir o cuidado de enfermagem no centro do processo gerencial ${ }^{(4)}$.

Essas inquietações suscitaram a realização da presente pes@uisa, cujo objetivo foi caracterizar as atividades gerenciais do enfermeiro em um Pronto-Socorro (PS) e Que corresponde ao recorte de uma investigação maior ${ }^{(8)}$.

\section{MÉTODO}

Tratou-se de um estudo Qualitativo desenvolvido em um hospital universitário filantrópico de Curitiba, PR, com oito enfermeiros do PS, cujo total desta categoria profissional corres-ponde a dez. Todos os enfermeiros do setor foram convidados a participar da pesquisa, porém dois deles se recusaram, o que determinou o numerário amostral. Dos peseuisados, um foi do turno da manhã, um atuava das sete às dezesseis horas e possuía função de coordenador do setor, dois eram do turno da tarde, dois da noite $A$ e dois da noite $B$.

A coleta dos dados foi realizada de fevereiro a abril de 2009 e foram respeitados os preceitos éticos estabelecidos pela Resolução $\mathrm{n}^{\circ}$. 196 de 10 de outubro de 1996 do Conselho Nacional de Saúde ${ }^{(9)}$. Deu-se após assinatura do Termo de Consentimento Livre e Esclarecido por parte dos pesquisados e aprovação do projeto 
pelo Comitê de Ética em Pesquisa da instituição hospitalar, registrado neste com o protocolo n'. 7799-08 e Certificado de Apresentação para Apreciação Ética (CAAE) o n. 0128.0.081.000-08.

Utilizou-se a técnica de entrevista semi-estruturada como opção investigatória, gravada com autorização dos sujeitos e abordando os seguintes Questionamentos: O Que você entende por atividade gerencial na atuação do enfermeiro? Quais as atividades gerenciais Que você desenvolve no seu processo de trabalho cotidiano?

As informações oriundas da coleta de dados foram tratadas por meio da análise de conteúdo ${ }^{(10)}$, composta de três etapas: a préanálise, a exploração do material e o tratamento dos resultados. Essa escolha deu-se devido à valorização do significado do conteúdo das mensagens de acordo com o objetivo proposto pela pesQuisa. Os resultados foram apresentados em forma de categorias exemplificadas com trechos dos discursos dos sujeitos e fundamentadas com a literatura pertinente.

\section{RESULTADOS E DISCUSSÃO}

Dos enfermeiros participantes da pesquisa dois foram do sexo masculino e seis feminino, sendo Que a média de idade foi de 29 anos. O Que trabalha há mais tempo no PS está há oito anos e o Que atua a menos tempo há dois anos, com média de 3,9 anos. Dentre os sujeitos, apenas dois já aturam no PS de outra instituição hospitalar.

Somente dois não possuem pós-graduação e os demais possuem lato-sensu, dos Quais três são especialistas em enfermagem em emergência e três especialistas em enfermagem em terapia intensiva.

A partir da análise de conteúdo emergiram duas categorias de análise: Gerência voltada para atender às demandas institucionais e gerência voltada para atender às demandas do cuidado de enfermagem.

\section{Gerência voltada para atender às demandas institucionais}

Esta categoria possui como subcategorias: ênfase em atividades burocráticas, gerenciamento de vagas, a administração do tempo ineficaz e sofrimento no trabalho.

A primeira subcategoria destaca a ênfase em atividades burocráticas voltadas à produtividade exigida pela instituição. As instituições hospitalares, assim como outras organizações, estão inseridas no modo de produção capitalista e, por conseguinte, apresentam-se arraigadas à necessidade de lucros oriundos dos serviços prestados à clientela. $\mathrm{O}$ enfermeiro Que atua nesse contexto incorporou ao longo dos tempos essa lógica ao seu modo de gerenciar, de maneira a atender às imposições institucionais galgadas em normas e rotinas centralizadas na lógica racional do trabalho ${ }^{(4)}$.

Assim, suas práticas administrativas distanciam-se progressivamente de finalidades confluentes ao cuidado de enfermagem e fazem uma aproximação mais intensa ao alcance das metas institucionais. Isso é decorrente de uma série de fatores, dentre os Quais a forte conotação empresarial conferida às instituições de saúde pelo célere processo de complexificação sofrido pelas mesmas, tanto no Que se refere à sua função específica de cura e promoção da saúde, Quanto na sua organização administrativa e diversificação dos profissionais Que aí atuam ${ }^{(1)}$.

Destarte, o enfermeiro pode deparar-se com certa frequência com disparidades no mundo do trabalho. Este fato foi relatado pelos sujeitos pesquisados, como pode ser observado nos seguintes trechos por eles mencionados:

Para mim o enfermeiro gerencia a todo o momento, porém, vejo que hoje, [...] ao adentrar ao mercado de trabalho é cobrado para assumir atividades administrativas de interesse da instituição, o Que faz com Que este profissional muitas vezes se coloque em posição de prevalecimento das atividades burocráticas. (EE2)

[...] Quando você me pergunta o que é atividade gerencial eu te respondo que depende. [...] se for o Que a instituição Quer que façamos, diz respeito a um monte de burocracia que poderia muito bem ser feita por outros profissionais Que não o enfermeiro. (EE3)

O gerenciamento do enfermeiro na prática clínica há tempos se fundamenta nas necessidades burocráticas e formais da organização, de modo a privilegiar os objetivos organizacionais em detrimento do cuidado de enfermagem propriamente dito, o que pode provocar tensões, desmotivação e descrenças no âmbito do trabalho ${ }^{(3)}$.

Estudos realizados em cenários distintos do pesquisado também identificaram essa problemática. Constatam, sobretudo, Que o exercício gerencial do enfermeiro tem-se caracterizado pela vinculação acentuada a normas preestabelecidas e configura-se uma prática reiterativa $^{(12-13)}$.

Os enfermeiros, ao realizarem o gerenciamento alicerçado em práticas burocráticas demandadas pela organização hospitalar, limitam-se a preocupar-se com a estrutura funcional da unidade em Que está lotado, o que o distancia da administração tendo o cuidado como foco ${ }^{(4)}$.

Ao serem Questionados sobre Quais atividades gerenciais desenvolvem no seu processo de trabalho cotidiano, um ponto nitidamente enfatizado pelos enfermeiros do PS em Questão, relacionado ao gerenciamento focado nas demandas da instituição, foi o fluxo inadequado de pacientes no setor. Externaram que frequentemente há estagnação dos pacientes internados após serem sanadas as necessidades emergenciais. Segundo os sujeitos, isto ocorre por falta de uma cultura institucional com vistas à otimização do serviço de emergência, no concernente ao gerenciamento de vagas, Que corresponde à segunda subcategoria.

Com relação a este problema, os participantes informaram Que, conforme normas estabelecidas pelo hospital, cabe ao enfermeiro procurar leitos disponíveis nas unidades de internamento para encaminhar aQueles indivíduos internados após atendimento no PS, como relatado nas seguintes falas:

[...] procuro vagas disponíveis nos demais setores com o intuito de proporcionar um melhor fluxo para o atendimento de emergência, apesar de que isso eu acho errado [...] (EE I)

Especificamente nesse PS, o enfermeiro ainda tem Que correr atrás de vagas para os pacientes internados, se não fizermos isso, superlota o setor e não temos mais como atender outras emergências. Mas isso porQue não há um bom serviço do cen- 
so hospitalar e temos Que ficar ligando em todos os setores para procurar leitos. Isso me revolta. (EE2)

[...] procuro vagas para pacientes internados no PS aguardando leito em outros setores. Isso para mim é o fim! Não sei para Que existe um censo hospitalar se não funcional...] (EE7)

O fluxo inadeeuado de pacientes internados já fora identificado por em um estudo realizado no mesmo $\mathrm{PS}^{(14)}$. Para as autoras, uma vez atendidos, os clientes Que precisam de internamento tendem a permanecer no serviço de emergência, impossibilitando outros atendimentos. Isso gera uma descaracterização deste serviço e, consequentemente, um desvio das funções emergenciais da eQuipe de enfermagem ${ }^{(14)}$.

A permanência de pacientes internados no PS não é privilégio do cenário em oue esta pesquisa foi desenvolvida. Com um fluxo de entrada contínuo e de saída lento, ocorre uma lotação excessiva, geralmente acima da capacidade do setor. Essa superlotação compromete a Qualidade do atendimento, independentemente das condições de trabalho disponíveis, agravando-se, sobremaneira, Quando tais condições não são as mais adeQuadas. Começa, então, uma seleção dos cuidados Que serão oferecidos ${ }^{(15)}$.

A presença desses pacientes é também alvo de contrariedade dos profissionais de enfermagem, Que se vêem obrigados a se desdobrarem para atender tantos indivíduos, Que "não são mais do seu setor", já Que não são mais casos de emergência. Além da evidente sobrecarga de trabalho, esses pacientes não motivam o profissional de enfermagem, uma vez Que não exigem dele conhecimentos específicos de urgência/emergência ${ }^{(15)}$.

A situação do fluxo deficitário dos pacientes internados no PS e da necessidade dos enfermeiros dedicarem-se à procura de leitos disponíveis em outros setores da instituição ficou evidente durante a coleta de dados. Em todas as entrevistas essa prática foi apontada, bem como um fator negativo advindo desta: a administração do tempo ineficaz, Que corresponde à próxima subcategoria. Tal observação é confluente com as colocações exaradas pelos sujeitos, como pode ser visto nos trechos a seguir:

[...] a gente perde um monte de tempo no plantão tentando achar leito para possibilitar outros atendimentos. (EE7)

Eu perco muito tempo no telefone procurando leitos disponíveis em outros setores. Deveria ter uma pessoa para viabilizar essas informações, um censo Que fosse confiável, o Que pouparia muito o enfermeiro do PS e este poderia se dedicar a outras atividades. (EE4)

Como o tempo é valioso e finito, aprender utilizá-lo com sabedoria requer tanto Qualidades de liderança como funções da administração. Trata-se de um elemento fundamental e determinante na produtividade do trabalhador, seja eual for a atividade por ele desempenhada, uma vez Que a produção é uma dimensão Que está vastamente presente no mundo do trabalho ${ }^{(16)}$.

Sendo assim, administrar o tempo é uma competência essencial a ser desenvolvida pelos enfermeiros, pois os mesmos devem se planejar para realizar todas as atividades diárias de cuidado aos pacientes, resolução de problemas dos funcionários, ações buro- cráticas exigidas pela instituição e ainda, terem tempo para os imprevistos. Desta maneira, a percepção do tempo como fator decisivo na organização do trabalho facilita a compreensão da relação deste com o processo de produção. ConseQuentemente, também auxilia a compreender o trabalho no processo de produção em saúde ${ }^{(17)}$.

Essas dificuldades gerenciais vivenciadas pelos enfermeiros pesQuisados podem servir como estopim para o sofrimento no trabaIho. Em um processo de trabalho cujos limites são a vida ou a morte de pessoas, como é o caso do pronto-socorro, na medida em Que as condições dadas se revelam precárias e o próprio profissional tenha de resolver problemas decorrentes da falta de racionalização desse processo de trabalho, o trabalhador poderá se revestir de uma 'couraça' composta de frieza e distanciamento para suportar o próprio sofrimento de 'saber o Que fazer', mas não 'ter as condições de fazer'. Essa frieza defensiva inevitavelmente significará desapego ao paciente ${ }^{(15)}$.

O trabalho nos serviços de urgência e emergência hospitalares pode ser satisfatório e fonte de prazer, mas, muitas vezes, cansativo e fonte de sofrimento ${ }^{(18)}$. Os trabalhadores da emergência deste estudo vivenciam diariamente prazer e sofrimento no trabalho, numa relação dialética, sendo Que a superlotação é um dos fatores Que causam maior sofrimento aos profissionais nesse cenário.

$\mathrm{O}$ aumento de recursos materiais, humanos e de espaço físico, não acompanhou o crescimento da complexidade nos serviços de urgência e emergência . Assim, os profissionais de saúde vêem-se confrontados com cargas elevadas de trabalho, com espaços físicos inadeeuados Que lhes causam sofrimento, conflitos e impossibilitam a expressão da subjetividade no trabalho ${ }^{(18)}$.

A ambivalência do exercício da gerência na enfermagem está no fato deste poder originar processos de alienação e também poder ser considerada um potente instrumento para a emancipação, aprendizado, experimentação, solidariedade e democracia. Portanto, apresenta-se como potencial causadora de sofrimento ou prazer no trabalho, de acordo com a postura tomada pelo enfermeiro ${ }^{(19)}$.

Se por um lado o enfermeiro inserido no PS Que serviu de cenário para este estudo necessita gerenciar com a finalidade de suprir as determinações institucionais, por outro, o cuidado de enfermagem é sua responsabilidade, como pregado pelas bases da profissão. Essa dimensão da gerência será abordada na categoria a seguir.

\section{Gerência voltada para atender às demandas do cuidado de enfermagem}

As subcategorias compositoras desta categoria são: o cuidado como foco da atividade gerencial, gerenciamento como influência positiva à assistência, o planejamento do cuidado de enfermagem e Sistematização da Assistência de Enfermagem (SAE) como ferramenta do gerenciamento.

A política de saúde vigente, as relações de poder, de saber, de agir entre diferentes profissionais, a tecnologia disponível e a complexidade dos casos atendidos são fatores Que influenciam, histórica e socialmente, no modo de gerenciar em enfermagem ${ }^{(19)}$.

Hodiernamente, os hospitais adouirem feições de uma empresa de saúde e valoriza intensamente o processo e os resultados financeiros. Esse viés positivista Que enfatiza a objetividade e a Quantificação em detrimento ao interesse pela clientela ecoa no processo de trabalho do enfermeiro ${ }^{(11)}$. 
Diante desta realidade, o enfermeiro defronta-se com dificuldades para entrelaçar os diferentes subprocessos ao cuidado, entre eles, o gerenciamento, pois a visão gerencial requerida do enfermeiro pela instituição diverge da construída ao longo de sua formação inicial.

Mesmo com tal obstáculo, os participantes do estudo reconhecem o cuidado como foco da atividade gerencial, cerne das ativi-dades administrativas desempenhadas pelo enfermeiro, conforme versam as falas a seguir:

Eu acredito que atividade gerencial é como um conjunto das atividades desempenhadas pelo enfermeiro para coordenar a assistência de enfermagem dentro do seu local ou campo de trabalho de maneira efetiva e eficaz, buscando sempre alinhar recursos físicos e humanos com a melhor assistência a ser oferecida ao paciente/cliente [...] Ainda Que eu faça um monte de burocracia, de alguma maneira isso vai colaborar de um jeito ou de outro para o cuidado das vítimas Que chegam no PS. Por isso que eu acho muito estranho Quando falam "eu sou só enfermeiro administrativo", porQue de alguma maneira esse trabalho vai refletir na assistência. (EEI)

Gerenciar é a capacidade de coordenar a assistência de enfermagem com eficiência, resolver os impasses Que surgem durante o dia de trabalho, planejar ações, avaliar a Qualidade da assistência prestada pela equipe, desenvolver educação continuada, planejar recursos humanos, controlar materiais e equipamentos sempre visando a melhor atenção ao paciente. (EE6)

A divisão do trabalho na enfermagem entre os diferentes profissionais Que a compõem apresenta condicionantes históricos e consolidou-se a partir das proposições nightingaleanas. Desde então, a cisão entre gerenciar e cuidar aparece com maior nitidez e permeia a profissão até a atualidade. Este dilema tem suas raízes na sistematização de conhecimentos da enfermagem, Que desde sua concepção apresenta uma dissociação entre teoria e prática, com predomínio de técnicas como ponto mais valorizado pelos profissionais ${ }^{(20)}$.

Há a necessidade de imbricação entre cuidar e administrar. Gerenciar em enfermagem pressupõe ter como objeto: os agentes do cuidado e os recursos empregados para a assistência; como agente: o enfermeiro; como instrumentos: bases ideológicas e teóricas de administração e prática de gerenciamento de recursos; finalidade: coordenar o processo de assistir em enfermagem; métodos: planejamento, tomada de decisão, supervisão e auditoria; produto: condições para o cuidado se efetivar com eficiência e eficácia ${ }^{(2)}$.

Assim como exposto os participantes da pesQuisa consideram Que não há cuidado possível se não houver a coordenação do subprocesso de trabalho cuidar em enfermagem, o Qual corresponde à finalidade do subprocesso de administrar.

Ainda nessa linha de pensamento, os sujeitos peseuisados pronunciam o gerenciamento como influência positiva à assistência e enfatizam a necessidade da consciência científica para tal prática, conforme constatado nas seguintes falas:

[...] o enfermeiro Que se acha somente assistencial também acaba fazendo a parte administrativa, às vezes até sem perceber, o
Que para mim é muito perigoso, pois administrar de maneira aleatória, sem consciência científica do Que se está fazendo, pode acabar influenciando negativamente na Qualidade do cuidado ao nosso paciente e também para a nossa equipe e o setor como um todo. (EEI)

Para mim, gerenciar é a parte mais importante de ser enfermeiro, porque dela depende todo o restante do sucesso do seu trabalho. Eu defino essa atividade gerencial como a área estratégica para organizar o processo de trabalho, tornando-o mais Qualificado tanto para o paciente Quanto para a equipe de enfermagem e a própria instituição. (EE5)

Como enfermeira de pronto socorro, durante meu processo de trabalho, eu procuro organizar o funcionamento do meu setor para Que ele possa ser capaz de oferecer uma assistência de Qualidade ao paciente. (EE7)

A cientificidade aparece na literatura como instrumento do gerenciamento $^{(2)}$, o Que vai ao encontro das falas anteriores. Destarte, a prática gerencial deve estar distante do empirismo e o seu produto é conferir condições para Que haja sucesso no cuidado de enfermagem. Reafirma-se, assim, a necessidade de o enfermeiro assumir o gerenciamento do cuidado e ressalta-se Que para coordenar as atividades necessárias para a sua produção é preciso conhecimento, responsabilidade e capacidade de decisão ${ }^{(21)}$.

Para isto, o planejamento do cuidado de enfermagem mostrase como função essencial para a efetividade deste processo e foi identificado como tal pelos enfermeiros do PS. Todos os entrevistados mencionaram o planejamento como importante sustentador da assistência.

Mesmo diante da instabilidade ligada à demanda típica de um pronto-socorro, durante a coleta de dados, ficou evidente a importância Que os sujeitos destinam ao ato de planejar. Atividades como elaboração de escalas, previsão de férias e cobertura de turnos com déficit de recursos humanos fazem parte do cotidiano desses profissionais como ferramenta para a busca do cuidado de enfermagem de Qualidade em um cenário de grandes adversidades.

Outro aspecto Que todos os sujeitos mencionaram nas entrevistas como atividade gerencial cotidianamente desenvolvida é a classificação do grau de dependência dos cuidados de enfermagem dos pacientes do setor.

A determinação do nível de complexidade dos pacientes no PS estudado é feita conforme o modelo proposto por Montezeli e Lopes ${ }^{(14)}$, segundo o Qual, os pacientes são divididos em duas cate-gorias: os internados sem leito ou em observação e aqueles atendidos na sala de emergência. A classificação dos internados ou em observação é feita uma vez ao dia, representando uma amostra. Todos aqueles que são atendidos na sala de emergência são classificados ao darem entrada no serviço, permitindo a identificação do total de atendimentos segundo a necessidade dos cuidados de enfermagem.

O sistema de classificação de pacientes permite sustentar o planejamento da assistência de enfermagem e mostra-se necessário para subsidiar o dimensionamento de recursos humanos e materiais, previsão dos custos da assistência e melhor distribuição de atividades entre os membros da equipe de enfermagem ${ }^{(22)}$.

Ainda na temática do planejamento do cuidado como função 
gerencial, alguns sujeitos citaram a SAE como ferramenta do gerenciamento.

No cenário da pesQuisa não há SAE completa, contudo, os enfermeiros realizam etapas do processo de enfermagem, principalmente o exame físico e a evolução dos pacientes em estado crítico Que permanecem no aguardo de leitos em Unidade de Terapia Intensiva. Esta constatação foi descrita como atividade gerencial apenas nas entrevistas de dois dos sujeitos: EE3, EE6.

A base de sustentação para a realização da SAE é o processo de enfermagem, o Qual pode ser entendido como instrumento para a implementação da assistência direta ao cliente. Este é constituído em fases ou etapas Que envolvem a identificação de problemas de saúde do cliente, delineamento do diagnóstico de enfermagem, realização da prescrição de enfermagem, implementação do cuidado e avaliação dos cuidados prestados, denominado de evolução de enfermagem ${ }^{(23)}$.

\section{CONSIDERAÇÕES FINAIS}

A trajetória percorrida permitiu o alcance do objetivo proposto pelo estudo e evidenciou Que os sujeitos vivenciam os dilemas pontuados pela literatura entre a ênfase burocrática do trabalho exigida pela organização e o gerenciamento voltado à assistência de enfermagem.

A cisão entre gerenciar e cuidar, bem como o gerenciamento realizado apenas com a lógica e o controle mecânico das ativi- dades, muitas vezes executadas por outros agentes, não encontra eco no perfil de enfermeiro exigido na atualidade. Assim, fazer o gerenciamento do cuidado implica tê-lo como pilar mestre das ações de enfermagem e fazer uso dos saberes administrativos e das novas tecnologias para realizá-lo. Para tal, faz-se necessário o envolvimento dos enfermeiros a fim de Que haja mudanças no fazer gestão do cuidado com criatividade e autonomia.

Sabe-se, porém, Que a grande maioria das instituições de saúde nas Quais o enfermeiro se insere está distante de alcançar tal realidade, o que dificulta a articulação da gerência ao cuidado e muitas vezes culmina no retrocesso ao cartesianismo e à escola clássica da Administração como fios condutores da prática deste profissional.

Os achados desta pesquisa propiciaram uma série de reflexões sobre a prática do enfermeiro de emergência e fornece subsídios, ainda Que embrionários, para desafios a este profissional. O princípio destes desafios ancora-se na articulação das diversas competências gerenciais em um cenário Que valoriza sobremaneira o aspecto técnico como elemento fundamental para uma prática produtivista. Vislumbramos Que os resultados possam instigar nas colegas deste serviço a inspiração necessária para uma transformação de sua prática.

Salientamos, contudo, Que existem limitações na pesQuisa pelo fato da mesma ter sido desenvolvida no PS de apenas uma instituição hospitalar. Sugerimos, portanto, ampliação do universo investigativo para outras realidades, uma vez Que a exploração da temática gestão em serviços de emergência está distante de ser sanada.

\section{REFERÊNCIAS}

1. Kirchhof ALC. O trabalho da enfermagem: análise e perspectivas. Rev Bras Enferm 2003;56(6): 669-73.

2. Sanna MC. Os processos de trabalho em enfermagem. Rev Bras Enferm 2007; 60(2): 221-24.

3. Trevisan MA, Mendes IAC, Shinyashiki GT, Gray GI. Gerenciamento do enfermeiro na prática clínica; problemas e desafios em busca de competência. Rev Latino-am Enfermagem 2006; 14(3): 457-60.

4. Ferraz CA. As dimensões do cuidado em enfermagem: enfoque organizacional. Acta Paul Enferm 2000; 13(esp): 91-7.

5. Fonseca MG, Rezende G, Alvarenga MF, Brasil SMC. Razões Que levam o enfermeiro a delegar atividades Que lhe são privativas. Enferm Atual 2003; 3(13): 12-8.

6. Wehbe G, Galvão CM. O enfermeiro de unidade de emergência de hospital privado: algumas considerações. Rev Latino-am Enfermagem 2001; 9(2): 86-90.

7. Peres AM, Ciampone MHT, Wolff LD. Competências gerenciais do enfermeiro nas perspectivas de um curso de graduação de enfermagem e do mercado de trabalho. Trab Educ Saúde 2008; 5(3): 453-72.

8. Montezeli JH. O trabalho do enfermeiro no pronto-socorro: uma análise na perspectiva das competências gerencias [dissertação]. Curitiba: Universidade Federal do Paraná; 2009.

9. Ministério da Saúde (BR). Conselho Nacional de Saúde. Resolução 196: sobre pesquisa envolvendo seres humanos. Brasília: Ministério da Saúde; 1996.

10. Bardin L. Análise de conteúdo. Lisboa: Edições 70; 1977.

11. Belltato R, Pereira WR. As potencialidades da enfermeira na

gestão do cuidado em saúde. Rev Bras Enferm 2003; 56(I): 6I-6.

12. Fernandes MS. A função do enfermeiro nos anos 90: réplica de um estudo. [dissertação] Ribeirão Preto: Escola de Enfermagem de Ribeirão Preto, Universidade de São Paulo; 2000.

13. Peduzzi M, Anselmi ML. O processo de trabalho de enfermagem: a cisão entre planejamento e execução do cuidado. Rev Bras Enferm 2002; 55(4):392-98.

14. Montezeli JH, Lopes AA. Implantação de um sistema classificatório do grau de dependência dos cuidados de enfermagem em um serviço de emergência. Cogitare Enferm 2006; I I(3): 239-44.

15. Deslandes SF. Violência no cotidiano dos serviços de emergência hospitalar: representações, práticas, interações e desafios [tese]. Rio de Janeiro: Escola Nacional de Saúde Pública, Fundação Oswaldo Cruz; 2000.

16. Marquis BL, Houston CI. Administração e liderança em enfermagem: teoria e prática. $4^{\mathrm{a}}$. ed. Porto Alegre: Artmed; 2005.

17. Mello MC, Fugulin FMT, Gaidzinski RR. O tempo no processo de trabalho em saúde: uma abordagem sociológica. Acta Paul Enferm 2007; 20(1): 87-90.

18. Almeida PJS, Pires DEP. O trabalho em emergência: entre o prazer e o sofrimento. Rev Eletrônica Enferm 2007; 9(3): 617-29.

19. Massaro M, Chaves LDP. A produção científica sobre gerenciamento em enfermagem hospitalar: uma pesquisa bibliográfica. Cogitare Enferm 2009; 14(1): 150-58.

20. Gonçalves AM, Sena RR. Assistir/cuidar na enfermagem. Rev Mineira Enferm 1998; 2(1): 2-8. 
Montezelli JH, Peres AM, Bernardino E.

21. Marques GQ, Lima MADS. Organização tecnológica do trabaIho em um pronto atendimento e a autonomia do trabalhador de enfermagem. Rev Esc Enferm USP 2008; 42(1): 41-7.

22. Carmona LMP, Évora YDM. Grau de dependência do paciente em relação à enfermagem: análise de prontuários. Rev Latinoam Enfermagem 2003; I I (4): 468-73.
23. Bittar DB, Pereira LV, Lemos RCA. Sistematização da assistência de enfermagem ao paciente critico: proposta de um instrumento para coleta de dados. Texto Contexto Enferm 2006: 15(4): $617-28$. 